\title{
A quite-small adenoid cystic carcinoma in a patient with parotid gland sialolithiasis
}

\author{
Masayuki Takano*1 ${ }^{* 1}$ Akira Watanabe ${ }^{1}$ and Kenichi Matsuzaka ${ }^{2}$ \\ ${ }^{1}$ Department of Oral and Maxillofacial Surgery, Tokyo Dental College, Misakicho 2-9-18, Chiyoda-ku, Tokyo, Japan \\ ${ }^{2}$ Department of Clinical Pathophysiology, Tokyo Dental College, Misakicho 2-9-18, Chiyoda-ku, Tokyo, Japan
}

\begin{abstract}
We present herein a rare case of a quite-small adenoid cystic carcinoma (AdCC) found coincidentally during an operation to remove a sialolith in the parotid gland. The patient was a 64-year-old man. The computed tomography revealed a sialolith-like radiopaque lesion in the left parotid gland. Chronic parotiditis and parotid gland sialolithiasis were diagnosed and an operation performed under general anesthesia to remove the sialolith. During removal of the $2 \times 6-\mathrm{mm}$ sialolith from the superficial layer of the parotid gland, a $3 \times 4-\mathrm{mm}$ hard opalescent mass was identified slightly posterior to the sialolith and removed concomitantly. Histopathological examination of the mass revealed an adenoid cystic carcinoma. Therefore, the superficial layer of the left parotid gland was excised as second surgery. The postoperative course is good, with no sign of local recurrence or pulmonary metastasis.
\end{abstract}

\section{Introduction}

Sialolithiasis, commonly encountered in a clinical setting and usually arising in the submandibular gland, is only relatively rarely found in the parotid gland [1]. Adenoid cystic carcinoma (AdCC) is a malignant tumor occurring in many types of tissue [2]. A report of malignant tumor of the salivary gland accompanying sialolithiasis is rare. We present herein a case of a previously undetected quite-small AdCC revealed during removal of a sialolith in the parotid gland.

\section{Case report}

The patient was a 64-year-old man who first visited our department with a swollen left parotid gland. At this time, discharge of pus was observed from the left parotid papilla. Although no abnormality was apparent on orthopantomography, a plain X-ray tangential to the parotid gland and computed tomography (CT) revealed a 2-mm radiopaque lesion with a clear border in the left Stensen's duct. The patient's personal and family medical histories revealed no relevant findings.

Based on a diagnosis of acute parotiditis and sialolithiasis, antibiotics were administered and the parotid papilla irrigated to alleviate inflammation. Although acute symptoms disappeared, a small amount of pus discharge persisted. Parotid gland swelling and pain occasionally recurred, and an operation was performed to remove the sialolith from an area slightly posterior to the parotid papilla via an intraoral approach under general anesthesia.

Despite subsequent alleviation of symptoms, swelling and pain recurred in the same manner in the left parotid gland one year after the surgery. CT revealed a further sialolith-like radiopaque lesion in the left parotid gland (Figure 1). Parotid gland irrigation was performed continuously and antibiotics administered when symptoms appeared. Four years later, subsequent CT revealed a sialolith-like radiopaque lesion increasing in size, and surgical extraction of the sialolith was scheduled. As the sialolith was located posterior to the superficial layer of the parotid gland, removal through a preauricular incision was planned.

\section{Clinical diagnosis}

Chronic parotiditis and parotid gland sialolithiasis

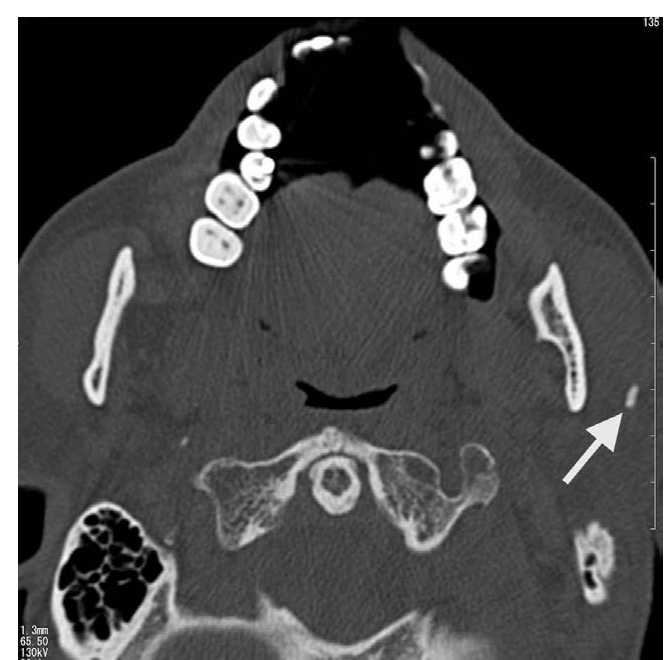

Figure 1. Axial CT image of sialolith-like radiopaque lesion in left parotid gland.

Correspondence to: Masayuki Takano, Division of Oral and Maxillofacial Surgery, Department of Oral and Maxillofacial Surgery, Suidobashi Hospital, Tokyo Dental College, Misakicho 2-9-18, Chiyoda-ku, Tokyo, Japan, Tel: 81-35275-1725; Fax: 81-3-3262-3213; E-mail: takano@tdc.ac.jp

Key words: adenoid cystic carcinoma, sialolithiasis, sialadenitis, parotid gland Received: January 07, 2017; Accepted: January 20, 2017; Published: January 23, 2017 


\section{Procedures and course}

An operation was performed under general anesthesia to remove the sialolith in the left parotid gland. Intraoperatively, isolation of the sialolith proved somewhat difficult, as the entire parotid gland was inflamed and hardened. However, a $2 \times 6-\mathrm{mm}$ sialolith was eventually removed from the superficial layer of the parotid gland. At this point, a $3 \times 4$-mm hard opalescent mass was identified slightly posterior to the sialolith and removed concomitantly (Figures 2 and 3 ). Histopathological examination of the mass revealed an adenoid cystic carcinoma. Therefore, the superficial layer of the left parotid gland was excised. Histopathological testing of the excised specimen showed no residual adenoid cystic carcinoma. At present, over nine years after the last surgery, the postoperative course is good, with no sign of local recurrence or pulmonary metastasis.

\section{Histopathological examination}

Histopathological analysis revealed that the excised specimen, a $3 \times 4$-mm opalescent tumor, contained a $1 \times 2$-mm mass of tumor tissue characterized by no clear borders and diffuse proliferation. Although tubular structures were observed in some areas, the degree of differentiation was low. And the surrounding tissue was characterized by strong hyalinization and basal membrane thickening. Tumor proliferation was mostly uniform, with no cribriform patterning (Figure 4). Immunohistological analysis revealed restiform proliferation of neoplastic myoepithelial cells strongly positive for vimentin and S-100. Although no completely cribriform structures were observed, cells showing intense staining for Ki-67 were (Figure 5). Based on these

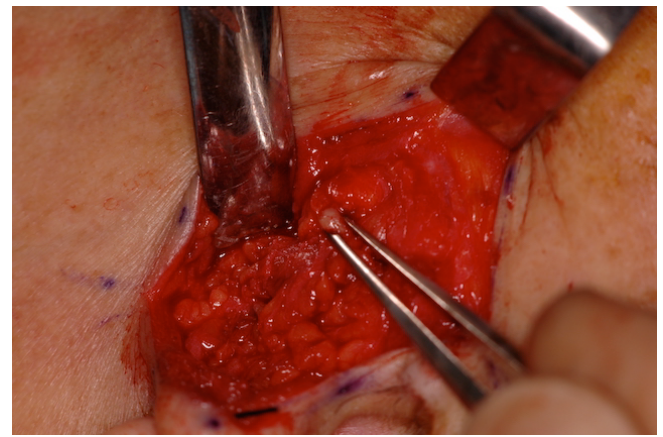

Figure 2. $3 \times$ 4-mm hard opalescent mass removed from site posterior tosialolith

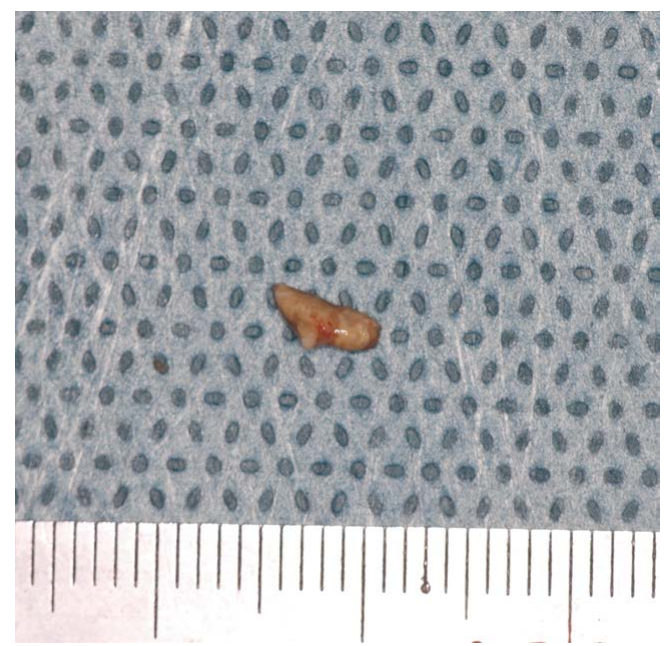

Figure 3. $2 \times 6$-mm sialolith removed from the superficial layer of the parotid gland.

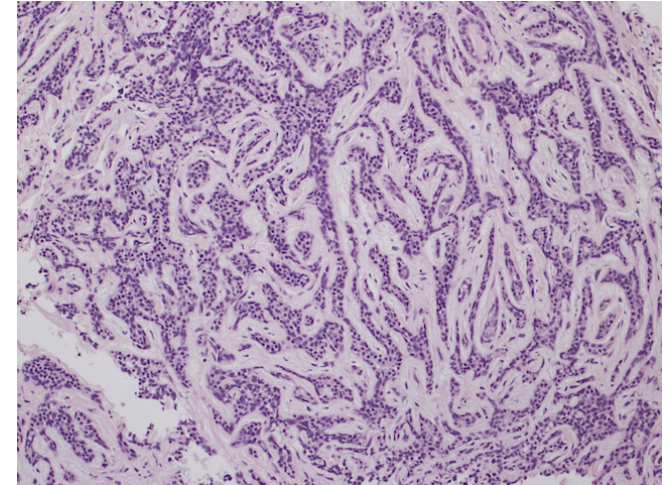

Figure 4. Tubular structures were observed in some areas, but low degree of differentiation. Tumor mostly proliferated uniformly with no cribriform patterning. (H-E stain).

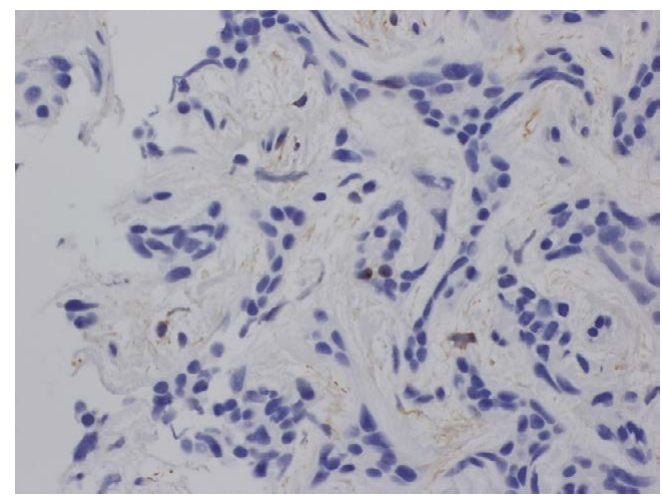

Figure 5. Cells showed intense staining for Ki-67 but no adenoid structures.

results, adenoid cystic carcinoma was diagnosed.

\section{Discussion}

Adenoid cystic carcinoma (AdCC) is a malignant tumor occurring in many types of tissue, including salivary gland. AdCC comprise approximately $10 \%$ of all epithelial salivary neoplasms and most frequently involve the parotid, submandibular and minor salivary glands. They comprise $30 \%$ of epithelial minor salivary gland tumors with the highest frequency in the palate, followed by the tongue, buccal mucosa, lip and floor of mouth. The tumor occurs in all age groups with a high frequency in middle-aged and older patients. There is no apparent sex predilection except for a high incidence in women with submandibular tumors $[1,2]$. In a recent report from Asian country, AdCC were more frequently sited in minor salivary glands (70\%) than in major glands. And the palate was the most common gland involved (31\%), the second most frequent sites were the parotid gland (12\%) [3].

In general, the clinical course of AdCC is long, with occasional local recurrence or metastases. AdCC proliferates invasively along nerves, and local recurrence is often caused by insufficient resection. Therefore, AdCC in the parotid gland requires total or superficial layer resection. Growth is gradual, and the duration between surgery and recurrence tends to be longer than in a normal malignancy. Even with a combination of surgery and radiotherapy, many patients experience recurrence and metastasis, and the 7 -year recurrence rate is believed to be over $70 \%[4,5]$.

Tumors are generally solid, grayish white, nodular and solitary, ranging in size from $2 \mathrm{~cm}$ to $5 \mathrm{~cm}$. Histopathologically, AdCC is characterized by relatively small, cubic or polygonal tumor cells 
resembling ductal epithelia and myoepithelial cells with eosinophilic cytoplasm. In typical AdCC, the parenchyma forms a cribriform lesion with microcystic cavities, although some tubular structures may be seen. Hyalinization of fibrous connective tissue is sometimes observed in the interstitium [1,2]. Low-grade pleomorphic adenocarcinoma and polymorphic adenoma need to be differentiated. In the present patient, no complete cribriform structures (a histopathological feature of AdCC) were observed, leading to a diagnosis of AdCC. In this case, some cells stained intensely for Ki-67, indicating a high level of cellular activity.

In the present patient, the quite-small adenoid cystic carcinoma was discovered coincidentally during an operation to remove a sialolith [6,7]. Some previous reports described a case of a large AdCC accompanying submandibular salivary gland sialolithiasis $[8,9]$. Hasegawa et al. reported a case of AdCC complicated with sialolithiasis of the submandibular gland [10]. The patient had noticed a swelling in her sublingual area for 10 years. The authors emphasized that the case indicated AdCC could arise in the background of chronic sialadenitis and suggested that long-standing sialadenitis cases should be carefully examined to exclude suspicion of malignancy before surgery. Shenoy et al. reported a case of co-existence presentation of AdCC with salivary calculi in the parotid and suggested that the malignancy could have developed because of chronic irritation by parotid calculi [11] On the other hand, a malignant salivary gland tumor accompanying sialolithiasis are extremity rear. Ide indicated that epithelial salivary gland tumor preceded by chronic obstructive sialadenitis is presently unavailable from epidemiologic data and careful assessment must be taken to ensure that neoplasm is secondary to inflammation [12]. The mechanisms underlying the onset of AdCC a remain to be clarified. Whether chronic local inflammation acts as a trigger for tumor expression is unclear, but this seems unlikely, as few reports have described sialolithiasis accompanying salivary gland tumor. Anyhow, we should be examine samples precisely after salivary gland excisions.

Papillary thyroid carcinoma found coincidentally after thyroidectomy is a well-known type of microcarcinoma [13]. The results of histopathological analyses have shown that the frequency of microcarcinoma is high. According to World Health Organization criteria, the maximum diameter is $10 \mathrm{~mm}$, and more than $90 \%$ lack clinical symptoms and go undetected. The incidence of cervical lymph node metastasis in papillary thyroid microcarcinoma is reportedly $50 \%-60 \%$. However, in major salivary gland, resection is usually performed for sialolithiasis or chronic sclerotic sialadenitis, but we can find few reports describing quite-small or micro carcinoma in such a case [14]. Malians reported a rare case of a 1-cm AdCC in the parotid gland that was resected after long-term facial pain [15]. The possibility of discovering a microcarcinoma may increase if detailed pathological examination of the entire salivary gland is performed after resection.

\section{References}

1. Neville BW, Damm DD, Allen CM, Chi AC (2016) Sialolithiasis (Salivary Calculi Salivary Stones). Adenoid cystic carcinoma. Oral and Maxillofacial Pathology. 4th ed. St. Louis: Saunders Elsevier. p.427-29: 462-64.

2. El-Naggar AK, Huvos AG, Barnes L, Eveson JW, Richard P, et al. (2005)Adenoid cystic carcinoma. Pathology and Genetics Head and Neck Tumours, World Health Organization Classification of Tumours. New York: WHO Publications Center. 221222

3. Tian Z, Li L, Wang L, Hu Y, Li J (2010) Salivary gland neoplasms in oral and maxillofacial regions: a 23 -year retrospective study of 6982 cases in an eastern Chinese population. Int J Oral MaxillofacSurg 39: 235-242.[Crossref]

4. Kim KH, Sung MW, Chung PS, Rhee CS, Park CI, et al. (1994) Adenoid cystic carcinoma of the head and neck. Arch Otolaryngol Head Neck Surg 120: 721-726. [Crossref]

5. Kokemueller H, Eckardt A, Brachvogel P, Hausamen JE (2004) Adenoid cystic carcinoma of the head and neck--a 20 years experience. Int J Oral MaxillofacSurg 33: 25-31.[Crossref]

6. Lustmann J, Regev E, Melamed Y (1990) Sialolithiasis. A survey on 245 patients and a review of the literature. Int J Oral MaxillofacSurg 19: 135-138.[Crossref]

7. Baurmash H, Dechiara SC (1991) Extraoral parotid sialolithotomy. J Oral MaxillofacSurg 49: 127-132.[Crossref]

8. Batzakakis D, Apostolopoulos K, Bardanis I (2006) A case report of coexistence of a sialolith and an adenoid cystic carcinoma in the submandibular gland. Med Oral Patol Oral Cir Bucal 11: E286-288.[Crossref]

9. Nanda KDS, Mehta A (2011)Sialolith and adenoid cystic carcinoma in the submandibular gland. A rare case. J ClinExp Dent 3: 251-255.

10. Hasegawa M, Cheng J, Maruyama S, Yamazaki M, Iida A, et al. (2011) Complication of adenoid cystic carcinoma and sialolithiasis in the submandibular gland: report of a case and its etiological background. Int J Oral MaxillofacSurg 40:647-650.[Crossref]

11. Shenoy VS, Kamath MP, Sreedharan S, Suhas SS (2015) Adenoid cystic carcinoma of the parotid gland associated with salivary calculi. An unusual presentation.J Cancer Res Ther 11:652.[Crossref]

12. Ide F (2011) Does calculus-related chronic sialadenitis represent a risk state for adenoid cystic carcinoma? Int J Oral MaxillofacSurg 40: 1449.[Crossref]

13. Sakorafas GH, Giotakis J, Stafyla V (2005) Papillary thyroid microcarcinoma: a surgical perspective. Cancer Treat Rev 31: 423-438.[Crossref]

14. Preuss SF, Klussmann JP, Wittekindt C, Drebber U, Beutner D, et al. (2007) Submandibular gland excision: 15 years of experience. J Oral MaxillofacSurg 65: 953957.[Crossref]

15. Malins TJ, Farrow A (1991) Facial pain due to occult parotid adenoid cystic carcinoma. J Oral MaxillofacSurg 49: 1127-1129.[Crossref]

Copyright: (C2017 Takano M. This is an open-access article distributed under the terms of the Creative Commons Attribution License, which permits unrestricted use, distribution, and reproduction in any medium, provided the original author and source are credited. 